\title{
Skull Metastasis of Gastric Gastrointestinal Stromal Tumor Successfully Managed by Surgery
}

\author{
Inkeun Park, M.D., 'Dong Hae Chung, M.D., Ph.D, ${ }^{2}$ Chan Jong Yoo, M.D., Ph.D., ${ }^{3}$ Dong Bok Shin, M.D., Ph.D.' \\ Division of Hematology-Oncology, Departments of Internal Medicine, Pathology, Neurosurgery, Gil Medical Center, Gachon University \\ College of Medicine, Incheon, Korea
}

Gastrointestinal stromal tumors (GISTs) are rare, but are the most common mesenchymal neoplasm of the gastrointestinal tract. The most common sites of metastasis are liver and peritoneum, while bone metastasis is rare. We report on a patient with skull metastasis after seven years of treatment with imatinib for metastatic GIST. She underwent metastasectomy consisting of craniectomy with excision of the mass, and cranioplasty and continued treatment with imatinib and sunitinib, without evidence of cranial recurrence. She died of pneumonia sepsis one year after metastasectomy. Skull metastasis of GIST is a very rare presentation, and an aggressive multidisciplinary approach should be considered whenever possible.

Key Words : Gastrointestinal stromal tumor · Skull metastasis · Metastasectomy · Target therapy · Multidisciplinary treatment.

\section{INTRODUCTION}

Gastrointestinal stromal tumors (GISTs) are rare, but are the most common mesenchymal neoplasm of the gastrointestinal tract, with an incidence of $10-20$ cases per million ${ }^{7}$.

These tumors can arise from anywhere in the gastrointestinal tract, and are thought to originate from interstitial cells of Cajal. The stomach is the most common site of origin (60$70 \%$ ), and the small intestine (20-25\%) and the large intestine (5-10\%) are other usual sites ${ }^{7}$. It can present as localized disease, however, metastasis at presentation occurs in up to $50 \%$ of cases. In addition, localized disease can recur even after curative surgery. The most common sites to which gas- trointestinal tumors metastasize are liver and peritoneum, while lung, bone, or lymph node metastases are rare ${ }^{13}$. Surgical removal is the only curative option for localized GIST. In cases of metastasis, systemic treatment is a main treatment modality. Before the imatinib era, there was no effective systemic treatment and survival of patients was usually poor. Discovery of a gain-of-function mutation in the c-kit protooncogene in GIST and development of imatinib, a tyrosine kinase inhibitor against the mutation, resulted in a dramatic change of the prognosis of this disease ${ }^{13)}$. As a consequence, median overall survival for metastatic GIST in the imatinib era is approximately five years ${ }^{14)}$. Due to introduction of very effective target agents, we now encounter metastases of GIST,

- Received: June 5, 2014 •Revised: July 31, 2014 •Accepted: August 6, 2014

- Address for reprints : Dong Bok Shin, M.D., Ph.D.

Division of Hematology-Oncology, Department of Internal Medicine, Gil Medical Center, Gachon University College of Medicine, 21 Namdong-daero 774beon-gil, Namdong-gu, Incheon 21565, Korea

Tel : +82-32-460-8371, Fax : +82-32-460-1754, E-mail : dbs@gilhospital.com

This is an Open Access article distributed under the terms of the Creative Commons Attribution Non-Commercial License (http://creativecommons.org/licenses/by-nc/4.0) which permits unrestricted non-commercial use, distribution, and reproduction in any medium, provided the original work is properly cited. 
which used to be regarded as unusual sites of metastasis.

Here report on a patient with skull metastasis of GIST after seven years of imatinib treatment who underwent successful metastasectomy.

\section{CASE REPORT}

Ten years before the skull metastasis occurs, a 50-year old female patient complaining of dyspepsia was diagnosed with a GIST on the high body posterior wall of the stomach. She underwent gastrectomy, and complete resection of the tumor without rupture was accomplished. The tumor measured $9 \times$ $7 \times 6 \mathrm{~cm}$ in size with a mitotic rate of 52/50 high power field (HPF), less than $10 \%$ of necrosis, clear resection margin, and no lymph node metastasis. On immunohistochemical (IHC) staining, CD34 and c-kit were positive, while smooth muscle actin (SMA) and S-100 were focally positive. At that time, neither mutation analysis nor adjuvant imatinib treatment was a routine practice; therefore, she was followed-up with regular examination without adjuvant treatment.

Nine years before the skull metastasis occurs, recurrence was observed in the abdominal wall and peritoneum. She started $400 \mathrm{mg} / \mathrm{day}$ of imatinib, and very good partial response (PR) was achieved and maintained. Although recurred GIST responded well to imatinib, she was suffering from grade 3 anorexia and lethargy, therefore, she took imatinib intermittently. The tumor grew during the imatinib-off period, but shrank again when she resumed imatinib treatment.
Her tumor was under control for seven years, until the peritoneal mass in the right lower quadrant showed progression and invasion of adjacent small bowel and ascending colon. She underwent debulking surgery (small bowel resection and reanastomosis, right hemicolectomy), and the mass measured $11 \times 8 \mathrm{~cm}$ in size with $10-13 / 50 \mathrm{HPF}$ and positive radial resection margin. After surgery, no gross mass was observed; however, the dose of imatinib was increased to $600 \mathrm{mg} / \mathrm{day}$. Approximately 16 months later, she found a lump on her head. The mass continued to grow, and CT scan of brain and abdomen which were taken three months later showed a large mass located in the parietal cranial vault suppressing adjacent brain and a small single liver metastasis (Fig. 1). Subsequent MRI revealed a heterogeneously enhancing mass of approximately $7.7 \mathrm{~cm}$ extent involving the right parietal bone, parietal scalp, and parietal convexity with a heterogeneous signal intensity on a T1/T2 weighted image, multiple signal void on a $\mathrm{T} 2$ weighted image, and fatty marrow signal loss with enhancement (Fig. 1). She underwent craniectomy with excision of the mass, cranioplasty, and hepatic resection. On surgical field, about $10 \times 10 \mathrm{~cm}$ sized yellowish, relatively soft mass originating from skull was compressing dura and brain parenchyma. Because mass adhered to the dura and invasion with seeding was grossly suspected, the bone, tumor, and dura were removed as a unit. Diagnosis of metastatic GIST was confirmed by pathologic examination (Fig. 2). She experienced left hemiparesis postoperative day 1 , and CT scan indicated intracerebral hemorrhage in the right temporoparietal lobe. This delayed subcortical hemorrhagic
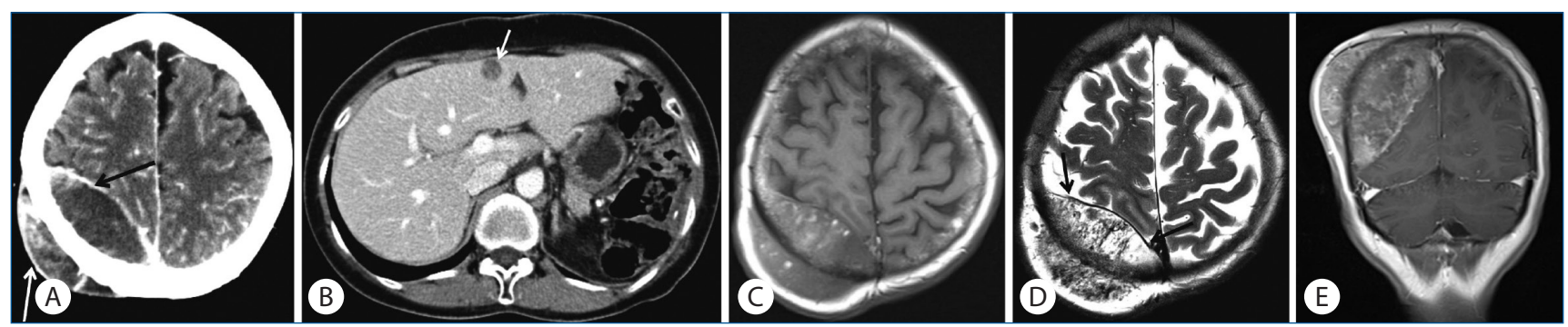

Fig. 1. Imaging findings of skull metastasis and hepatic metastasis in a patient with malignant gastrointestinal stromal tumor. A : Post-contrast brain CT image reveals a $7.7 \mathrm{~cm}$ sized, well-defined mass (arrows) centered on the diploic space of the calvaria. B : Abdomen CT image reveals a hypodense single metastasis (arrow) in left medial segment of the liver. C and D : Axial T1- (C) and T2-weighted (D) MR images show heterogeneous signal intensity of the skull metastasis. The dura (arrows) is intact and the underlying cortex appears compressed. Note the multiple signal voids on T2-weighted image. E : After gadolinium enhancement, the mass demonstrates heterogeneous enhancement. 

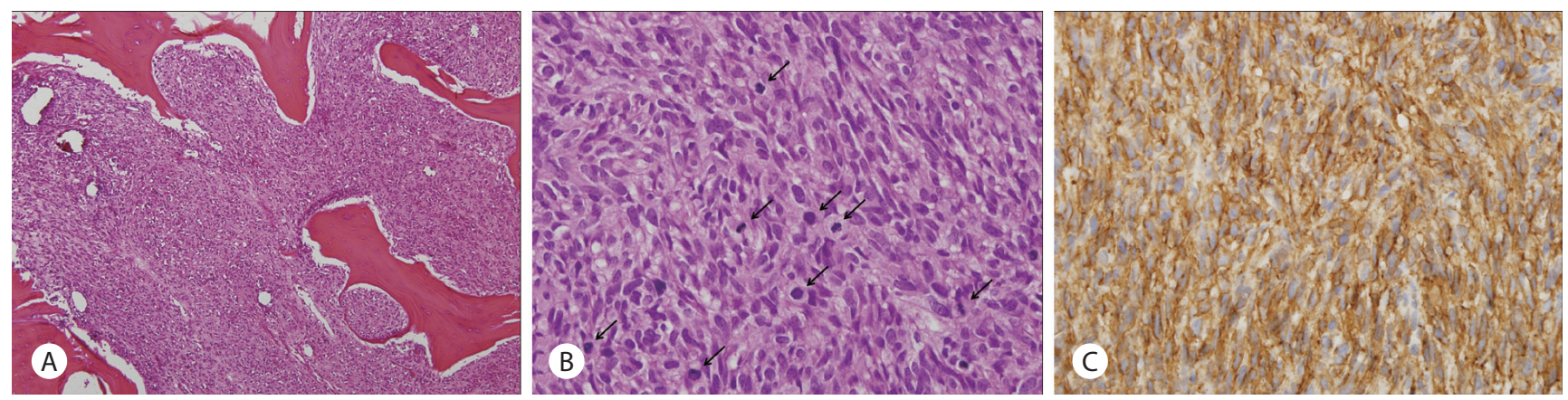

Fig. 2. Pathology of a resected skull tumor. A : Metastatic gastrointestinal stromal tumor in the skull. The tumor consists of atypical spindle cells with high cellularity and infiltrative growth pattern with destruction of normal bone tissue $(H \& E, \times 100)$. B : High power view of the tumor. Mitotic figures (arrows) are frequently noted $(\mathrm{H} \& \mathrm{E}, \times 400)$. C : Tumor cells are positive for c-kit (c-kit immunostain, $\times 400)$.

transformation was thought to result from drainage vein sacrifice during the operation. After conservative management and rehabilitation, she recovered and was discharged from the hospital. Because the role of adjuvant radiotherapy for GIST is not established, we did not administer adjuvant radiotherapy. Instead, her imatinib dose was elevated to 800 $\mathrm{mg} /$ day thereafter, which she was not able to tolerate. After three months of rest, huge masses were found in the liver and peritoneal cavity. There was no recurrence in the cranium. She started sunitinib treatment 6 months after metastasectomy. Unfortunately, she was not able to tolerate sunitinib due to nausea and fatigue; therefore, after 1.5 month of sunitinib treatment, she rested for three months. As the masses progressed, she resumed sunitinib, when 1 year has passed since metastasectomy. A month after sunitinib resumption, she was admitted to the hospital via the emergency department for fever and dyspnea, and diagnosed with pneumonia. She died of acute respiratory distress syndrome one month later, when 14 months has passed since skull metastasectomy.

\section{DISCUSSION}

This case illustrates a woman who had skull metastasis of imatinib-resistant GIST, which was treated by surgical removal. She lived for one year after craniectomy without evidence of cranial recurrence, and her cause of death was not disease progression itself but infection and respiratory failure.

Although malignant GISTs can theoretically metastasize any organ, they obviously have some tropism for specific organs. They tend to stay in the abdominal cavity even in very advanced stage ${ }^{133}$. Only a few papers have reported bone metastases of GIST ${ }^{1,5,6,8,10)}$, and the most frequent site of bone metastases reported were spine and pelvis ${ }^{5,6}$. Skull metastasis is even rarer. To the best of our knowledge, only five cases of skull metastasis have been reported ${ }^{2,3,9,11,15)}$. Presenting symptoms depended on the location of metastasis, with skull base or periorbital metastases being more symptomatic ${ }^{2-4,11,15)}$.

Management of metastatic GIST should be customized according to symptom, location of disease, available treatment modalities, and condition of the patient. Our patient underwent debulking craniectomy, because we believed that previous long-term imatinib treatment produced a resistant clone, a huge metastatic mass would soon provoke neurologic symptoms, and en-bloc resection would be possible. Debulking surgery is a viable option even in patients with metastatic GIST, especially when disease status is stable or shows limited progression ${ }^{12)}$. If a resistant clone is removed completely, long term survival could be achieved. Our patient survived 10 years after recurrence. Considering that median overall survival of metastatic or recurrent GIST receiving imatinib treatment is five years and nine-year overall survival rate is $34 \%{ }^{14)}$, she lived for a comparatively long period after recurrence. We believe that appropriate metastasectomy (peritoneal, hepatic, and skull metastasis) during the treatment course had an important role in her long-term survival.

Traditionally, bone metastasis of GIST was thought to be very rare; however, long-term survival of our patient enabled 
the disease to metastasize to an uncommon site. Likewise, with the much prolonged survival of GIST patients by virtue of effective target agents and multidisciplinary approach, medical and surgical oncologists will encounter more and more unusual presentations and complications of this disease. For resistant clones, local treatment such as surgery or radiofrequency ablation has a critical role. Therefore, identification of rare but complicated metastasis is essential for clinicians in order to ensure prompt administration of a precise treatment modality.

\section{CONCLUSION}

We present the case of a female with skull metastasis of GIST after a long term treatment with imatinib successfully managed by metastasectomy. Symptomatic skull metastasis from GIST is very rare, and an aggressive multidisciplinary approach including surgery and chemotherapy plays an important role in its management.

\section{References}

1. Abuzakhm SM, Acre-Lara CE, Zhao W, Hitchcock C, Mohamed N, Arbogast $D$, et al. : Unusual metastases of gastrointestinal stromal tumor and genotypic correlates : case report and review of the literature. J Gastrointest Oncol 2 : 45-49, 2011

2. Akiyama K, Numaga J, Kagaya F, Takazawa Y, Suzuki S, Koseki N, et al. : Case of optic nerve involvement in metastasis of a gastrointestinal stromal tumor. Jpn J Ophthalmol 48 : 166-168, 2004

3. Baeg MK, Bae SH, Lee KH, Kim J, Park IS, Jin JY : Diplopia as a presenting symptom in a gastric gastrointestinal stromal tumor. Jpn J Clin
Oncol 41 : 265-268, 2011

4. Barrière J, Thariat J, Vandenbos F, Bondiau PY, Peyrottes I, Peyrade F : Diplopia as the first symptom of an aggressive metastatic rectal stromal tumor. Onkologie 32 : 345-347, 2009

5. Bertulli R, Fumagalli E, Coco P, Messina A, Morosi C, Dileo P, et al. : Unusual metastatic sites in gastrointestinal stromal tumor (GIST). J Clin Oncol (Meeting Abstracts) 27 : 10566, 2009

6. Di Scioscio V, Greco L, Pallotti MC, Pantaleo MA, Maleddu A, Nannini M, et al. : Three cases of bone metastases in patients with gastrointestinal stromal tumors. Rare Tumors 3 : e17, 2011

7. Eisenberg BL, Pipas JM : Gastrointestinal stromal tumor--background, pathology, treatment. Hematol Oncol Clin North Am 26 : 1239 1259, 2012

8. Feki J, Bouzguenda R, Ayedi L, Bradi M, Boudawara T, Daoud J, et al. : Bone metastases from gastrointestinal stromal tumor : a case report. Case Rep Oncol Med 2012 : 509845, 2012

9. Gil-Arnaiz I, Martínez-Trufero J, Pazo-Cid RA, Felipo F, Lecumberri MJ, Calderero $\mathrm{V}$ : Skull metastasis from rectal gastrointestinal stromal tumours. Clin Transl Oncol 11 : 625-627, 2009

10. Jati A, Tatl $S$, Morgan JA, Glickman JN, Demetri GD, Van den Abbele A, et al. : Imaging features of bone metastases in patients with gastrointestinal stromal tumors. Diagn Interv Radiol 18 : 391-396, 2012

11. Li LF, Tse YH, Ho SL, Yan KW, Lui WM : Duodenal GIST metastasized to skull and orbit managed by surgery : a case report. Asian J Surg 34 : 181-184, 2011

12. Raut CP, Posner M, Desai J, Morgan JA, George S, Zahrieh D, et al. : Surgical management of advanced gastrointestinal stromal tumors after treatment with targeted systemic therapy using kinase inhibitors. J Clin Oncol 24 : 2325-2331, 2006

13. van der Zwan SM, DeMatteo RP : Gastrointestinal stromal tumor : 5 years later. Cancer 104 : 1781-1788, 2005

14. von Mehren $M$, Heinrich MC, Joensuu $H$, Blanke CD, Wehrle $E$, Demetri $\mathrm{GD}$ : Follow-up results after 9 years (yrs) of the ongoing, phase II B2222 trial of imatinib mesylate (IM) in patients (pts) with metastatic or unresectable KIT+ gastrointestinal stromal tumors (GIST). J Clin Oncol 29 (Suppl) : Abstract 10016, 2011

15. Wong CS, Chu YC : Intra-cranial metastasis of gastrointestinal stromal tumor. Chin Med J (Engl) 124 : 3595-3597, 2011 